\title{
A Transmission Range Reduction Scheme for Power-Aware Broadcasting in Ad Hoc Networks Using Connected Dominating Sets
}

\author{
Jie $\mathrm{Wu}$ \\ Dept. of Computer Science and Engineering \\ Florida Atlantic University \\ Boca Raton, Florida 33431 \\ Email: jie@cse.fau.edu
}

\author{
Bing Wu \\ Dept. of Computer Science and Engineering \\ Florida Atlantic University \\ Boca Raton, Florida 33431 \\ Email: bwu@cse.fau.edu
}

\begin{abstract}
In ad hoc wireless networks, due to node mobility, broadcasting is expected to be more frequent to update global route/network information. A straightforward broadcasting by flooding is usually very costly and will result in substantial redundancy and large energy consumption. Power consumption is an important issue since most mobile hosts operate on battery. Broadcasting based on a connected dominating set (CDS) is a promising approach, where only nodes in the CDS need to relay the broadcast message. A set is dominating if all the nodes in the system are either in the set or neighbors of nodes in the set. In general, nodes in the CDS consume more energy to handle various bypass traffic than nodes outside the set. To prolong the life span of each node and, hence, the network, nodes should be alternated when possible to form a CDS. In this paper, we further extend the power-aware broadcasting by dynamically reducing the transmission range of each node during the broadcast process without sacrificing the broadcast coverage. The effectiveness of the proposed methods in prolonging the life span of the network is confirmed through simulation.
\end{abstract}

\section{INTRODUCTION}

An ad hoc wireless network (or simply ad hoc network) is a special type of wireless network in which a collection of mobile hosts with wireless network interfaces may form a temporary network, without the aid of any established infrastructure or centralized administration. Examples of such networks are used in military, disaster rescue, wireless conferences, and monitoring in environment which are dangerous, remote and inaccessible in nature.

The dynamic and infrastructureless nature of ad hoc networks poses a special challenge in designing efficient broadcasting. Many papers discussed the broadcast issue in ad hoc networks [4], [6], [9], [10], [15]. A straightforward broadcasting by flooding (labeled as FL), where each node forwards the message once, is usually very costly and will result in substantial redundancy and large energy consumption. Dominating-set-based broadcasting [5] is based on the concept of connected dominating set (CDS) in graph theory. A subset of the nodes is a dominating set if every node not in the subset is adjacent to at least one node in the subset. This approach limits the broadcast process to a subgraph induced from the dominating set and simplifies the decision of retransmission

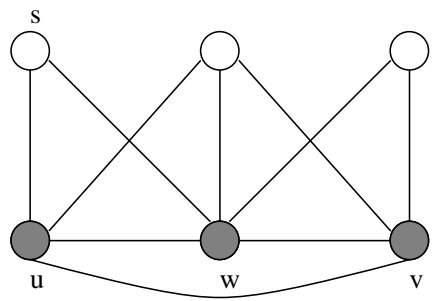

Fig. 1. Example of marking process

to the one in a smaller subnetwork. Since nodes outside the CDS do not need to forward the message, this mechanism will reduce power consumption, redundant retransmission and, hence, network contention. Clearly, the efficiency of this approach depends largely on the process of finding a CDS and the size of CDS. Unfortunately, finding a minimum CDS is NP-hard for most graphs [1].

$\mathrm{Wu}$ and $\mathrm{Li}$ [3] proposed a simple localized algorithm, called marking process, that can quickly determine a CDS in a given connected graph. A node is included in the CDS if two neighbors are not directly connected (see Figure 1 where gray nodes are nodes in the CDS). Wu and Li [3], [11] also proposed localized rules to reduce the size of CDS: if the neighbor set of node $u$ in the CDS is covered by that of another node $v$ (or those of two connected nodes $v$ and $w)$ in the CDS, then $v$ can be removed from the CDS. In this case, $u$ is said to be covered by $v$ (or by $v$ and $w$ ). To avoid simultaneous removal of two nodes covering each other, each node $u$ is assigned a distinct id, $i d(u)$, as the priority value. A node is removed from the CDS when it is covered by node(s) with higher id(s) (labeled as ID). In Figure 1, if $i d(w)=\max \{i d(u), i d(v), i d(w)\}$, both $u$ and $v$ can be removed; if $i d(w)=\min \{i d(u), i d(v), i d(w)\}, w$ can be removed.

The limitation of power of each host poses a unique challenge for power-aware design [12]. There has been an increasing focus on low cost and reduced node power consumption in ad hoc networks. Even in standard networks such as IEEE 


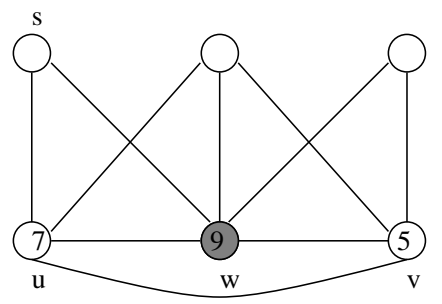

Fig. 2. Examples of extended energy rules

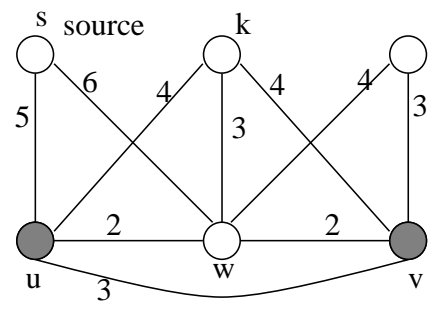

Fig. 3. Examples of extended distance rules

802.11, requirements are included to sacrifice performance in favor of reduced power consumption [13]. Saving the total power of each broadcast is not sufficient. A particular node may be depleted quickly if it is frequently selected as a gateway host by the marking process. In order to prolong the life span of each node and, hence, the network, power consumption should be minimized and balanced among nodes. Unfortunately, nodes in the dominating set in general consume more energy in handling various bypass traffic than nodes outside the set. Therefore, a static selection of dominating nodes will result in a shorter life span for certain nodes, which in turn results in a shorter life span of the whole network.

In [7], an energy cost model is given for transmitting and receiving operation. Receiving cost includes electronics parts while transmitting cost includes electronics parts and amplifier parts. Therefore, a transmitting operation costs more than a receiving operation. In Wu, Wu and Stojmenovic's [14] paper, it is assumed that gateway nodes and non-gateway nodes consume $d$ and $d^{\prime}$ units of power in a given update interval, respectively. $d$ and $d^{\prime}$ are variables which depend on network topology and traffic patterns. Specifically two extended rules are proposed in [14]; one is based on node degree (ND) ( $w$ can be removed in Figure 1) as the priority, and the other is based on energy level (EL) associated with each node (see Figure 2 , where the number inside each cycle is the energy level). The latter one, belonging to power-aware design [2], tries to prolong the life span of each node by balancing the energy consumption in the network. Under this rule, nodes are alternated when possible to form a CDS based on their residual energy levels, assuming nodes in the CDS consume more energy to handle various bypass traffic than nodes outside the CDS.

In this paper, we extend the power-aware broadcasting by dynamically reducing the transmission range of each node during the broadcast process without sacrificing the broadcast

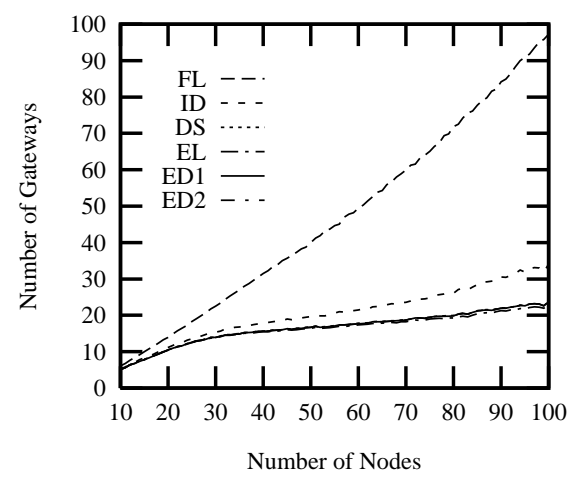

Fig. 4. Average number of gateway nodes for different schemes

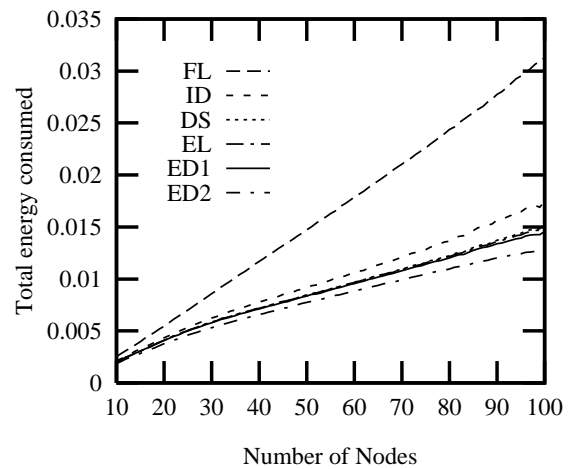

Fig. 5. Total power consumption per packet when the first node dies

coverage. It is assumed that each node knows the distance information of each neighbor (not positional information) through signal strength of "hello" messages. The energy cost model used is similar to the one in [7], [8] and is distancesensitive. Also, it is assumed that initially the network is connected under the uniform transmission range. Each node dynamically reduces its transmission range when possible based on neighbor distance information and the neighbor set of each neighbor or just the forwarding neighbor.

\section{Distance-Aware Rules}

The following extended rules are all localized aiming to reduce energy consumption of each node without sacrificing

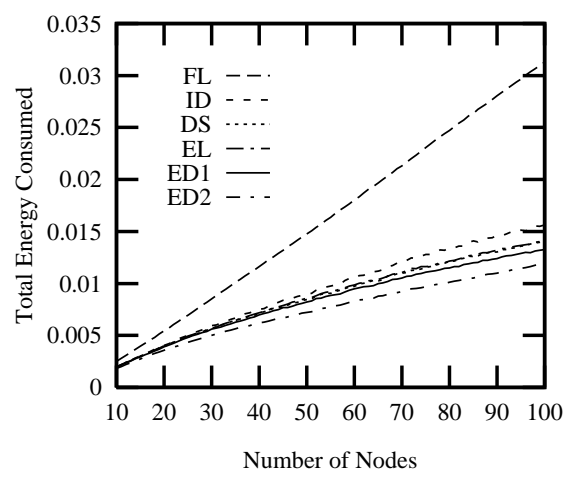

Fig. 6. Total power consumption per packet when $50 \%$ of nodes die 


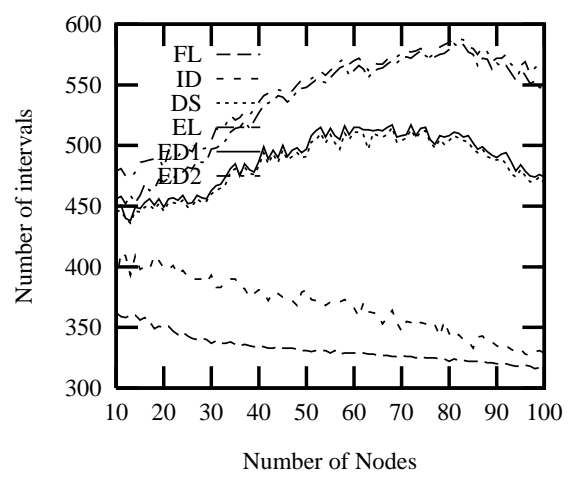

Fig. 7. Number of intervals when the first node dies

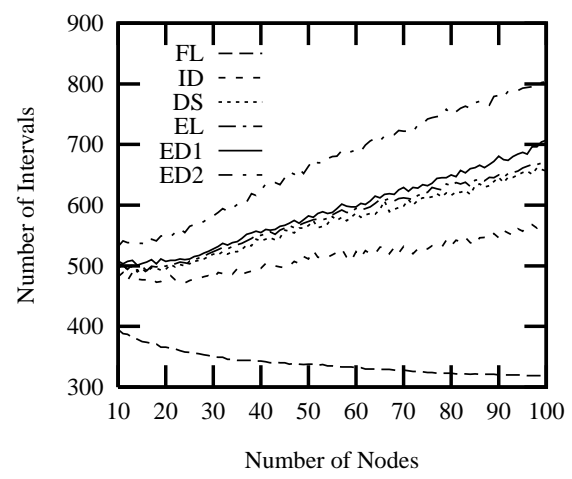

Fig. 8. Number of intervals when $50 \%$ of nodes die

the broadcast coverage. The maximum distance $(d s)$ is the distance from a node to all its neighbors.

Distance-aware rules (DS). When $u$ is covered by $v$, node $u$ can be removed from the CDS if $d s(u)>d s(v)$. Id is used to break a tie when $d s(u)=d s(v)$. Also, when $u$ is covered by two connected nodes $v$ and $w$, and additionally, $d s(u)=$ $\max \{d s(u), d s(v), d s(w)\}, u$ can be removed. In Figure 3, where distance information is associated with each edge, since $d s(u)=5, d s(v)=4$, and $d s(w)=6, w$ is removed from the CDS.

Distance-aware source-unrelated rules (ED1). First apply the marking process and DS rules to obtain the CDS. The $d s(u)$ is adjusted when $u$ in the CDS relays a broadcast packet. Here, $d s(u)$ is defined as $\max \{$ distance $(u, v) \mid v$ : neighbor of $u$, but not a neighbor of $w$, where $w$ is a neighbor of $u$ in CDS with $i d(w)>i d(u)\}$. In Figure 3, assuming that the uniform transmission range is 6 , the transmission range of $v$ can be reduced to 3 if $i d(u)>i d(v)$, since $v$ is no longer required to cover $k$.

Distance-aware source-related rules (ED2). Same as ED1, except $d s(u)$ is defined as $\max \{\operatorname{distance}(u, v) \mid v$ : neighbor (excluding the forwarding neighbor) of $u$, but not a neighbor of $w$, where $w$ is a neighbor of $u$ in CDS with $i d(w)>i d(u)$ \}. ED2 uses information related to a specific broadcasting. In Figures 3, if $s$ is the source and it is the forwarding neighbor for $u$, the transmission range of $u$ can be reduced to 4 , since $u$ does not need to cover $s$ and $w$.

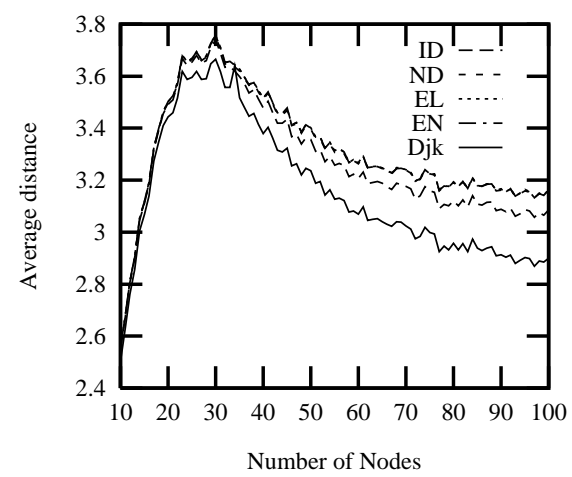

Fig. 9. The average distance for different schemes

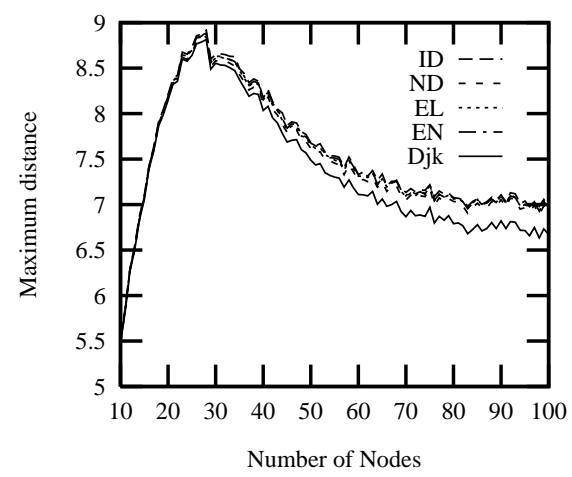

Fig. 10. The maximum distance for different schemes

\section{Performance Evaluation}

We measure the size of CDS after applying different rules. In addition, the average life span of the network, measured as the time the first depleted node occurs under different rules, is also simulated. The simulation is conducted in a $100 \times$ 100 2-D free-space by randomly allocating a given number of hosts ranging from 10 to 100 . The initial node transmission range is 25 . A unit disk graph is randomly generated, where node connections depend on node distances. The initial energy level is $1 J$. We assume that each node can adjust transmission range. The following energy consumption model is used:

1) send operation: $\alpha+\beta d^{k}$,

2) receive operation: $\alpha$

where $\alpha=50 \mathrm{~nJ} / \mathrm{bit}, \beta=100 \mathrm{pJ} / \mathrm{bit} / \mathrm{m}^{2}$, and $k=2 . d$ is the distance between the sender and receiver; the packet size is 2000 bits long. So a gateway node needs to consume power of $2 \alpha+\beta d^{k}$ when it relays each packet (1 bit) to its neighbors, but when the gateway is the source node it only consume $a+\beta d^{k}$ for each packet. For the non-gateway node, it consumes power $\alpha$ if it is not the source node. Otherwise it will also need power $\alpha+\beta d^{k}$ for each packet.

The simulation is divided into intervals, with one random broadcast in each interval. Four sets of data are collected (before the occurrence of the first depleted node): average size of CDS, total energy consumed, average number of intervals, average distance and maximum distance from the source to a destination (hop count). The simulation is conducted in a fixed 
network but can be easily extended to a dynamic one, since all rules are localized, that is, dependent on neighborhood information only.

Figure 4 shows results of the first simulation. The size of CDS under DS and EL stays very close. DS, ED1 and ED2 have the same size since all of them use the maximum distance as the priority. Figures 5 and 6 show results of the second simulation for life span until the first node and $50 \%$ of nodes deplete respectively. The figures show the total energy consumption of all nodes per broadcast. The number of depleted nodes doesn't affect the total power consumption per broadcast since the curve of these two figures are very similar. The (increasing) order of energy consumption is the reverse order of the life span of the first depleted node. In ED2, the node always consumes the least energy, followed closely by ED1.

Figures 7 and 8 show results of the third simulation. It is obvious that the total number of intervals before the first node dies should be less than intervals before $50 \%$ of the nodes die. Figures 7 shows that the (increasing) order of life span of the first depleted node is: FL, ID, DS, ED1, EL and ED2. The life span under FL is the shortest because it has the largest size of CDS. DS and ED1 stay very close. ED2 always has the longest life span. The number of intervals until the occurrence of the first depleted node is extended by about $45 \%-70 \%$ under these extended rules. Figures 7 shows that the increasing order of life span before $50 \%$ of the nodes deplete is: FL, ID, DS, EL, ED1 and ED2. In ED1 rule, the network has longer life span than under EL rule where the percentage of dead nodes increase. The number of intervals until the $50 \%$ of the nodes deplete is extended by about $52 \%-98 \%$.

Figures 9 and 10 show results for the forth simulation. Figure 9 shows the average distance from source to destination, Figure 10 shows the maximum distance from source to destination. Djk represents the shortest path derived by applying the Dijkstra's shortest path algorithm. Average distance of EL and DS are very close to each other with an average of 3.32 hops. FL and Djk are close with an average of 3.15 hops. ID is between the above two groups with an overall average distance of 3.27 hops. The maximum distance for NR and Djk is very close with an overall average of 7.36 hops. The maximum distance of ND and EN is also close to each other with an overall average of 7.55 hops. For ID and EL, their maximum distance stays close with an overall of average 7.53 hops.

\section{CONCLUSION}

In this paper, we have applied three extended rules for energy-aware broadcasting in ad hoc networks. In the broadcasting process, only dominating hosts are responsible for retransmitting the broadcast packet. The objective is to devise a selection scheme (for dominating hosts) so that the overall energy consumption is balanced in the network, and at the same time, a relatively small connected dominating set is generated. A simulation study has been conducted to compare the life span of the network under different methods. The distanceaware rules are the winners in terms of the long network life span. Experiments also show that all rules introduce little transmission latency in terms of the average and maximum number of hops. Our future work will perform more in depth simulation under different settings.

\section{ACKNOWLEDGMENT}

This work was supported in part by NSF grant CCR 9900646 and grant ANI 0073736.

\section{REFERENCES}

[1] T. W. Haynes, S. T. Hedetniemi, and P. J. Slater. Fundamentals of Domination in Graphs. Marcel Dekker, Inc., 1998.

[2] S. Singh, M. Woo, and C. S. Raghavendra. Power-aware routing in mobile ad hoc networks. Proc. of MobiCom'98. Oct. 1998.

[3] J. Wu and H. Li. On calculating connected dominating set for efficient routing in ad hoc wireless networks. Proc. of the 3rd Int'l Workshop on Discrete Algorithms and Methods for Mobile Computing and Communications (Dial M), 1999.

[4] C.-C. Chiang. Routing in clustered multihop, mobile wireless networks with fading channels. Proc. of IEEE SICON'97, pages 197-211, 1997.

[5] I. Stojmenovic, M. Seddigh, and J. Zunic. Internal node based broadcasting algorithms in wireless networks. Proc. of the 34th Annual IEEE Hawaii Int'l Conf. on System Sciences. 2001.

[6] B. Chen, K. Jamieson, H. Balakrishnan, and R. Morris. Span: An energy-efficient coordination algorithm for topology maintenance in ad hoc wireless networks. Proc. of MobiCom'01. July 2001.

[7] T. S. Rappaport. Wireless Communications. Prentice Hall, 1996.

[8] I. Stojmenovic and X. Lin. Power-aware localized routing in wireless networks. IEEE Transactions on Parallel and Distributed Systems. Vol. 12, No. 10, Oct. 2001.

[9] A. Qayyum, L. Viennot, and A. Laouiti. Multipoint relaying: An efficient technique for flooding in mobile wireless networks. RR-3898, INRIA, 2000, www.inria.fr/RRRT/RR-3898.html.

[10] C. K. Toh. Maximum battery life routing to support ubiquitous mobile computing in wireless ad hoc networks. IEEE Communications Magazine. 39, 6, June 2001, 138-147.

[11] J. Wu. Extended dominating-set-based routing in ad hoc wireless networks with unidirectional links. IEEE Transactions on Parallel and Distributed Systems. 3, 9, Sept. 2002, 866-881.

[12] C. Rohl, H. Woesner, and A. Wolisz. A short look on power saving mechanisms in the wireless LAN standard draft IEEE 802.11. Porc. of 6th WINLAB Workshop on Third Generation Wireless Systems, 1997.

[13] IEEE Standards Departments. IEEE draft standard - Wireless LAN. IEEE Press, 1996.

[14] J. Wu, B. Wu, and I. Stojmenovic. Power-Aware Broadcasting and Activity Scheduling in Ad Hoc Wireless Networks Using CDS. Proc. of IASTED Int'l Conf. on Wireless and Optical Communication (WOC). 2002.

[15] S. Y. Ni, Y. C. Tseng, Y. S. Chen, and J. P. Sheu. The broadcast storm problem in a mobile ad hoc network. Proc. of Mobicom 1999. 1999, 151-162. 